\title{
Polaron-induced lattice distortion of (In,Ga)As quantum dots by optically excited carriers
}

\author{
S. Tiemeyer, ${ }^{1, \text { * }}$ M. Bombeck,${ }^{2}$ M. Paulus, ${ }^{1}$ C. Sternemann, ${ }^{1}$ J. Nase,${ }^{1}$ H. Göhring, ${ }^{1}$ F. J. Wirkert,${ }^{1}$ \\ J. Möller, ${ }^{1}$ T. Büning, ${ }^{1}$ O. H. Seeck, ${ }^{3}$ D. Reuter, ${ }^{4}$, † A. D. Wieck, ${ }^{4}$ M. Bayer, ${ }^{5}$ and M. Tolan ${ }^{1}$ \\ ${ }^{1}$ Fakultät Physik / DELTA, Technische Universität Dortmund, D-44221 Dortmund, Germany \\ ${ }^{2}$ Experimentelle Physik II, Technische Universität Dortmund, D-44221 Dortmund, Germany \\ ${ }^{3}$ Deutsches Elektronen-Synchrotron DESY, D-22607 Hamburg, Germany \\ ${ }^{4}$ Angewandte Festkörperphysik, Ruhr-Universität Bochum, D-44780 Bochum, Germany \\ ${ }^{5}$ Experimentelle Physik 2, Technische Universität Dortmund, D-44221 Dortmund, Germany
}

(Dated: July 1, 2014)

\begin{abstract}
We report on a high resolution x-ray diffraction study unveiling the effect of carriers optically injected into (In,Ga)As quantum dots on the surrounding GaAs crystal matrix. We find a tetragonal lattice expansion with enhanced elongation along the [001] crystal axis that is superimposed on an isotropic lattice extension. The isotropic contribution arises from excitation induced lattice heating as confirmed by temperature dependent reference studies. The tetragonal expansion on the femtometer scale is attributed to polaron formation by carriers trapped in the quantum dots.
\end{abstract}

PACS numbers: 68.65.Hb, 61.05.cp, 63.20.kk, 71.38.-k

Self-assembled (In,Ga)As quantum dots (QDs) are crystalline inclusions on the scale of ten nanometers that are embedded in a GaAs matrix. Carriers residing in QDs are three-dimensionally confined, giving rise to discrete energy levels, similar to atoms. The excellent optical quality of such systems has allowed studies on fundamental problems of light-matter-interaction and has paved also the way of QDs into applications as light emitters, ranging from single photon sources to high-power laser diodes [1 4]. An important related problem with multiple facets is the interaction of carriers with phonons, which facilitates carrier relaxation into the ground states after non-resonant excitation [5] or mediates also coupling to the optical modes of a resonator [6]. On the other hand, this interaction sets limitations to the coherence of confined charge [7] or spin excitations [8].

Despite of their high relevance, carrier-phonon interactions are still not understood in full detail. Theoretically, over the years more and more elaborated models have been developed to account for experimental observations, for an overview see [5]. The models range from weak coupling pictures based on (modified) Fermi's golden rule, involving single- and two-phonon emission events 9 -12, to strong coupling descriptions leading to polaron formation and involving quantum kinetic effects [13-15. Typically, the carrier-phonon interaction has been assessed through intra- or interband optical transitions in QDs. [16. 17. For example, non-linear time-resolved methods like four-wave mixing revealed a drop of coherent exciton polarization on a few ps-time scale. [7 Through the temperature dependence of this drop, it could be uniquely related to the interaction with acoustic phonons. In the spectral domain, this results in spectral wings on both sides of the zero-phonon exciton spectral line [7, 18.

However, the impact of the coupling onto the lattice in form of a possible distortion [18] has not been directly and quantitatively assessed so far in experiment. For optical excitation resonant with the ground state transition the following dynamics were predicted theoretically [19]: A quasi-stable polaron, which is a bound state of the injected carriers and acoustic phonons, is formed, altering the lattice structure and changing the lattice constant. As a result of this distortion, a coherent phonon wave packet is emitted from the QDs, escaping on ps-time scale into the surrounding material. Due to loss of coherence by scattering, this wave packet eventually contributes to heating. For non-resonant excitation, carrier relaxation towards the ground state by phonon emission leads to additional lattice heating [20, 21].

(In,Ga)As QDs result from heteroepitaxial, strain driven Stranski-Krastanov growth 22, 23. The QDs are coupled to the surrounding GaAs matrix, thereby elastically deforming the lattice. Consequently, carrier-phonon interactions inside the QDs translate also into the GaAs crystal lattice. Here we study the GaAs distortion due to optically excited QD carriers by high-resolution x-ray diffraction (XRD), from which we obtain direct, quantitative evidence for the polaron-induced lattice expansion. While optically induced changes of bulk systems were considered already by x-ray analysis [26, this has not been achieved so far for nanostructures, requiring particular resolution and sensitivity. The XRD experiments were performed at the beamlines BL9 24] and P08 [25] of the synchrotron radiation facilities DELTA (TU Dortmund) and PETRA III (Deutsches ElektronenSynchrotron DESY), respectively.

The study was performed on an (In,Ga)As/GaAs QD multilayer structure grown on a (001) oriented GaAs substrate. After deposition of a $500 \mathrm{~nm}$ thick GaAs buffer layer, InAs corresponding to a nominal thickness of 1.9 monolayers was deposited at a substrate temperature of $525^{\circ} \mathrm{C}$, resulting in the formation of the wetting layer and 

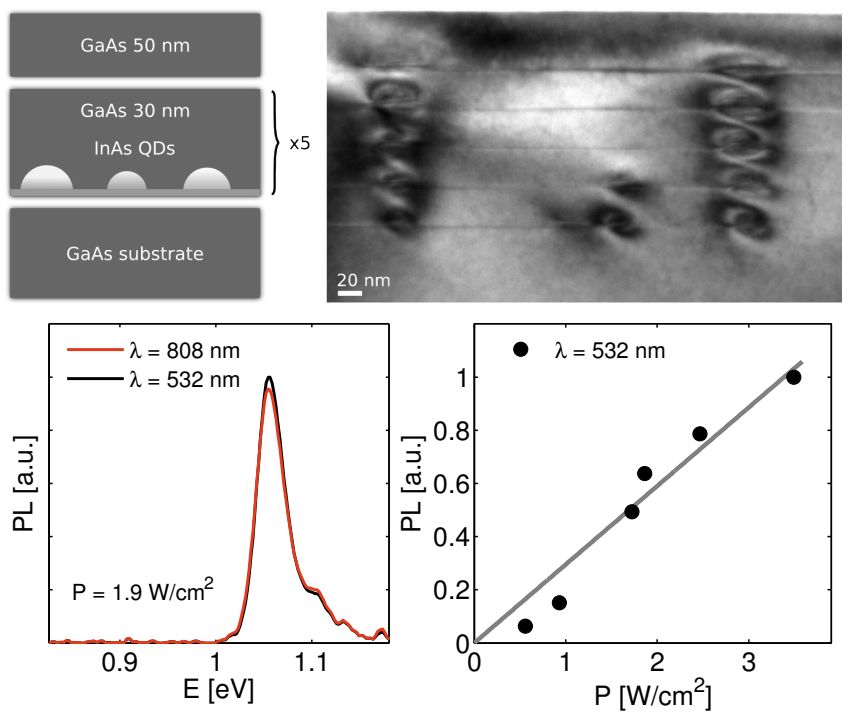

FIG. 1: Sketch and X-TEM image of the QD sample (top). PL spectra of the QD sample using excitation laser wavelengths of $532 \mathrm{~nm}$ and $808 \mathrm{~nm}$, respectively (bottom left). Linear dependence of PL intensity on laser power density (bottom right).

the dots. The QD layer was capped by a $30 \mathrm{~nm}$ GaAs spacer. This sequence was identically repeated five times. The last of these layers was capped with $50 \mathrm{~nm}$ GaAs. The top part of Figure 1 shows a sketch of the sample structure and a corresponding dark field cross-sectional transmission electron microscope (X-TEM) image. The $\mathrm{X}$-TEM image displays the stacks of lens-shaped QDs distorting also the surrounding matrix. The dot density is about $10^{10} \mathrm{~cm}^{-2}$. The QD size is approximately $8 \mathrm{~nm}$ and $30 \mathrm{~nm}$ in height and diameter, respectively. The sample was mounted onto the cold finger of a liquid helium continuous-flow cryostat. The sample temperature was $100 \mathrm{~K}$, large enough to avoid carrier localization in the wetting layer, but too small to lead to thermal carrier emission from the strongly confined ground states ( $0.4 \mathrm{eV}$ total confinement potential).

The setup developed for simultaneous optical excitation and XRD probing of the QD sample is shown in Figure 2 (top). Optical excitation was done by a diodepumped, continuous wave Nd:YAG laser supplying radiation at $532 \mathrm{~nm}$ wavelength with $1 \mathrm{~W}$ maximum power. The laser power on the sample was adjusted by a variable attenuator. The diameter of the laser spot on the sample surface was enlarged in order to obtain homogeneous illumination of the whole sample with size 5 by 5 $\mathrm{mm}^{2}$. Alternatively, a laser diode emitting at $\lambda=808 \mathrm{~nm}$ wavelength could be used for excitation with a maximum power of $0.6 \mathrm{~W}$. Both lasers excite electron-hole pairs into the GaAs barriers, however, with distinctly different energies in excess of the band gap.

Photoluminescence from the sample as result of the op- tical excitation was collected by a pair of two achromatic lenses and analyzed by a USB spectrometer. The bottom part of Figure 1 shows photoluminescence spectra recorded with either of the two lasers at the same excitation power while performing structural analysis, resulting in comparable emission intensities. Thus, comparable excitation powers lead to similar carrier densities in the dots. In the applicable power range, the intensity scales linearly with power, suggesting that the number of excited electron-hole pairs per dot remains below unity. A laser shutter system was added to the set-up, triggered by the beamline control. Thereby we could record diffraction curves of the sample, both optically excited and non-excited, within a single XRD scan by measuring each data point twice, once with opened and once with closed laser shutter.

The left part of Figure 2 (bottom) shows a reciprocal space map (RSM) in the vicinity of the GaAs(002) Bragg reflection for the non-excited QD sample, recorded at $\mathrm{T}=100 \mathrm{~K}$. The $\mathrm{x}$-ray photon energy was $12.38 \mathrm{keV}$. At this energy, the penetration depth of the x-rays [27] is in the $\mu$ m-range, so that effects occuring within the InAs QD multilayer structure, located in the top $0.2 \mu \mathrm{m}$ layer of the sample, become accessible.

The data are scaled to reciprocal lattice units with respect to the GaAs cubic lattice constant of $a_{\mathrm{s}}=0.565 \mathrm{~nm}$ at $T=300 \mathrm{~K}$. Along the $L$-direction in the RSM, the GaAs(002) Bragg reflection is accompanied by superlattice peaks originating from the layered structure of the QD sample. An analysis of these features by simulations utilizing the kinematical approximation connects the superlattice peaks to the periodic wetting layer/spacing layer system of the sample. However, as shown by the simulations, the QDs do not contribute significantly to the x-ray scattering pattern and can not be clearly resolved by the XRD measurements, in particular because the dot inhomogeneities lead to a strong broadening. On the other hand, changes by carriers trapped in the QDs affect also the surrounding matrix, so that insight into a possible carrier-induced lattice distortion may be taken from a GaAs reflection.

The middle part of Figure 2 gives the difference of two RSMs, one recorded for the excited $\left(I_{\mathbf{e}}\right)$ and one for the non-excited $\left(I_{\mathbf{n e}}\right)$ QD sample within a single scan. The difference scattering pattern exhibits a considerable shift of the $\mathrm{GaAs}(002)$ Bragg reflection as well as comparably small changes of the superlattice peak intensities. To classify the reliability of our measurements, the experiment was repeated but without optical excitation, as shown on the right side of Figure 2 The difference pattern $I_{\text {ne }}-I_{\text {ne }}$, now being a measure for the systematical errors of the experiment, identifies only the shift of the Bragg reflection as genuine effect of the optical excitation, whereas the altered intensities of the superlattice peaks are predominantly due to statistical fluctuations. A typical peak shift observed for a QD sample in the 

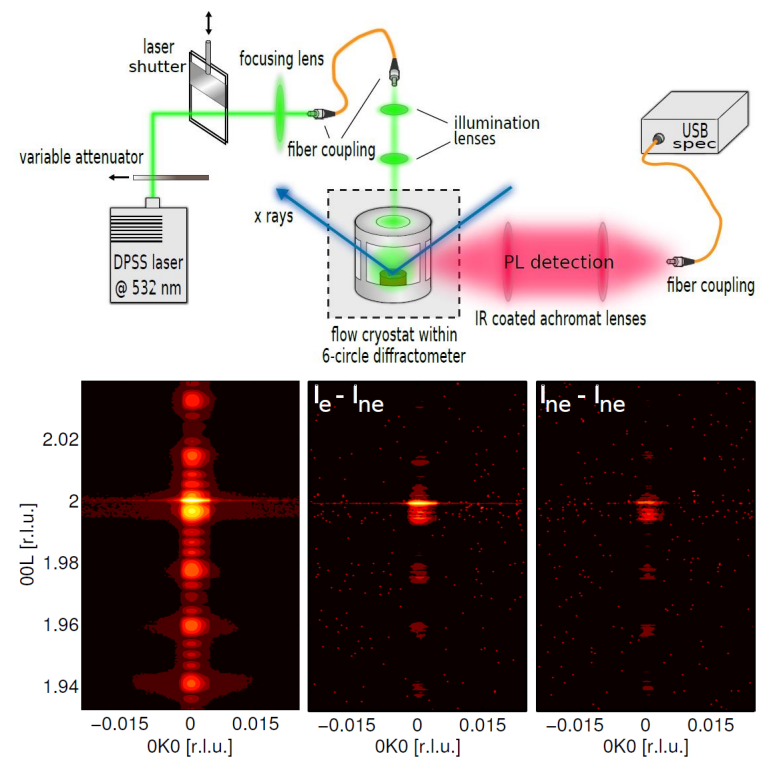

FIG. 2: Top: Schematic drawing of the experimental set-up. Bottom: (left) RSM of the QD sample recorded in the vicinity of the GaAs(002) reflection. (middle) Difference of two RSMs taken for excited and non-excited sample, respectively. (right) Reference difference of two RSMs measured each in non-excited state.

excited and non-excited state is shown in Figure 3 (top left).

The shift of the $\mathrm{GaAs}(002)$ Bragg reflection corresponds to an increase of the lattice constant of the GaAs matrix along the heterostructure growth direction. To elucidate the origin of this change, we studied the thermal expansion of the QD sample and, serving as a reference, of a (001) oriented bulk GaAs sample of comparable dimensions. We estimated possible laser excitation induced lattice heating effects by monitoring the energy of GaAs-related emission lines, namely of the band gap as well as defect-related transitions. Within the experimental accuracy of about $1 \mathrm{meV}$ we did not resolve a shift of these lines, limiting possible crystal temperature increase to $10 \mathrm{~K}$ over the whole applied excitation power range. For comparison, the thermal expansion of both samples was determined by measuring the change of lattice constant $a$ along the [001] crystal direction from the $\mathrm{GaAs}(002)$ and $\mathrm{GaAs}(004)$ Bragg reflections in the temperature range between $90 \mathrm{~K}$ and $125 \mathrm{~K}$ around the nominal sample temperature. These measurements were performed with a laboratory diffractometer in $\theta-\theta$ geometry, which was equipped with an x-ray tube emitting $\mathrm{Cu} \mathrm{K}{ }_{\alpha}$ radiation of $8.048 \mathrm{keV}$ photon energy. The change of the perpendicular lattice constant $\Delta a / a_{\mathrm{s}}$ normalized by the $300 \mathrm{~K}$ lattice constant is shown in Figure 3 (top right). We performed a linear regression to the data in order to evaluate differences in the thermal expansion of the two samples (see dashed line for QD [001] and solid line for GaAs [001]) and found, that the thermal lattice
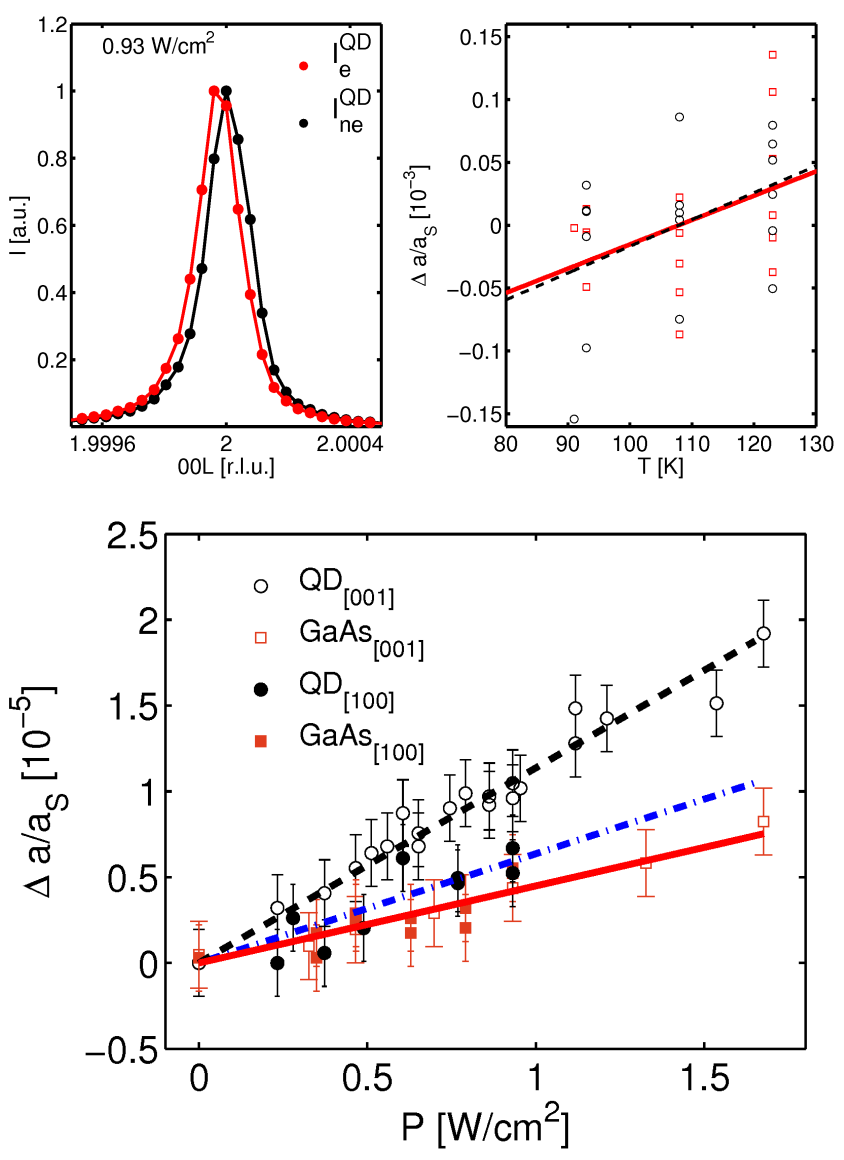

FIG. 3: Peak shift of the QD (002) Bragg reflection for the excited and non-excited state at a laser power of $0.93 \mathrm{~W} / \mathrm{cm}^{2}$ (top left). Comparison of thermal expansion in [001] direction of QD (open circles) and GaAs (open squares) reference sample with the temperature varied in the range between $90 \mathrm{~K}$ and $125 \mathrm{~K}$ (top right). The solid and dashed lines correspond a linear regression to the QD and GaAs data, respectively. Dependence of relative lattice expansion along [001] and [100] crystal directions on laser excitation power density for the QD sample and GaAs reference (bottom) together with linear fits to the QD data for the [001] crystal direction (dashed), QD data in [100] direction (dashed dotted), and GaAs data in [001] and [100] directions (solid).

expansion is hardly modified by the inclusion of the QDs. The change in slope of the linear fits for GaAs and QD sample is found to be $2 \cdot 10^{-7} \mathrm{~K}^{-1}$ and relates to a lattice constant difference between GaAs and QD sample in the order of $2 \cdot 10^{-6}$ for $\Delta T=10 \mathrm{~K}$.

Next we investigated the lattice expansion of the optically excited samples along the [001] and [100] crystal directions as function of laser power density $P$, where we first focus on the $532 \mathrm{~nm}$ illumination. For that purpose high-resolution XRD measurements of the $\operatorname{GaAs}(002)$ and $\operatorname{GaAs}(200)$ Bragg reflections were analyzed, also at $T=100 \mathrm{~K}$. The measurement of the $\operatorname{GaAs}(200)$ reflection was performed under a grazing incidence angle of $\alpha_{\mathrm{i}}=0.5^{\circ}$. The relative change of lattice constant, 
$\Delta a / a_{\mathbf{s}}(P)$, was obtained from determining the laserinduced shift of the corresponding Bragg reflection. The results of these measurements are shown in Figure 3 (bottom). Note that the excitation power densities are corrected for the reflectivities in the setup.

For the GaAs sample, identical linear dependencies of the relative lattice expansion on laser excitation power density are found for the [001] and [100] crystal directions. The solid line Figure 3 (bottom) shows the corresponding fit to the data. Hence, the laser irradiation of the GaAs reference causes an isotropic lattice expansion, as for thermal heating.

In contrast, the QD sample is characterized by a strongly anisotropic lattice expansion, very different from the expected isotropy of a pure thermal effect: while the excitation-induced expansion along the [100] direction (dshed-dotted line) is similar to that in the GaAs sample, it is considerably enhanced along the [001] direction (dashed line). The crystal therefore undergoes a tetragonal lattice distortion.

The data indicate that the in-plane effect occurs mostly from thermal lattice expansion. On the other hand, the enhanced expansion along the [001] direction apparently cannot be assigned to a sole thermal effect. This is further corroborated by power dependent measurements performed with the red laser emitting at $808 \mathrm{~nm}$ wavelength, corresponding to a photon energy of $E=1.53 \mathrm{eV}$. The mechanism of lattice heating is quite different then, because the carriers are excited only about one optical phonon energy (36.7 meV in GaAs) above the GaAs band gap $\left(E_{\mathrm{g}, \mathrm{GaAs}}=1.50 \mathrm{eV}\right.$ at $\left.T=100 \mathrm{~K}\right)$, while for the green laser with $E=2.33 \mathrm{eV}$ photon energy the excess energy of $0.8 \mathrm{eV}$ corresponds to more than 20 optical phonons. Once these energies have been released, carriers are trapped in the wetting layer or highly excited QD states. From there, further phonon emission has to occur to bridge the $0.4 \mathrm{eV}$ energy required for relaxation into the dot ground states, where the photoluminescence shown in Fig. 1 is generated. For red light excitation, the crystal heating therefore arises mostly from the QDs, while for green illumination the heating occurs more homogeneously in the crystal. Still we find for red light excitation that the lattice expansion along the [001] direction is significantly larger in the QD sample than for the GaAs reference. The independence of the tetragonal QD lattice distortion on laser excitation wavelength supports that it has an origin different from heating. 28]

We therefore suggest that the anisotropic contribution to the lattice distortion is mostly induced by the optically injected carriers after relaxation into their ground states. This relaxation occurs on a timescale of a few ten ps. After about 0.5 ns electrons and holes recombine radiatively, giving rise to the photoluminescence in Fig. 1. However, the continuous wave excitation maintains an on average steady carrier population in the quantum dot ensemble. The electron-hole pairs couple strongly to the lattice, leading to polaron formation.

This suggestion has to be tested regarding compatibility with the experimental findings. Most importantly, there is the anisotropy of the tetragonal lattice distortion. This anisotropy is in good accordance with the expectation from the distribution of the electron and hole wave functions in the QDs. From former studies, it is established that there is a mismatch of the corresponding charge distributions with the electron wave function located below that of the hole along the vertical growth direction of (In, Ga)As self-assembled quantum dots [29]. This implies that electron-hole pairs resemble electric dipoles oriented along the [001] crystal direction. The dipole orientation obviously facilitates and amplifies the polaron formation along this direction.

Next, there is the excitation power dependence. The QD population by electron-hole pairs in the ensemble is stochastic so that we monitor an average distortion of the lattice by the polaronic effects. With increasing excitation power, more QDs become populated by carriers and contribute to the lattice distortion along the [001] direction leading to an expansion increasing linearly with excitation power, similar to the increase of photoluminescence emission intensity. As function of excitation power it can be described by

$$
\Delta a / a_{\mathrm{s}}(P)=(0.5 \pm 0.12) \cdot 10^{-5} \cdot P
$$

where the laser excitation power $P$ is measured in $\mathrm{Wcm}^{-2}$. From this average distortion one may estimate the optically induced deformation at the QD layers. For simplicity, we assume this distortion to be homogeneous in the different layers. An important boundary condition is that we do see a shift of the X-ray reflections but no significant change in peak intensity and width: this limits the local strain differences to about $10^{-4}$. Together with the average lattice expansion on the order of $2 \mathrm{fm}$ per $\mathrm{W} / \mathrm{cm}^{2}$ and the exponential x-ray penetration profile into the sample with an absorption depth in the $\mu \mathrm{m}$ range, we estimate an overall lattice distortion of $5 \mathrm{fm}$ per $\mathrm{W} / \mathrm{cm}^{2}$ along the [001] direction at the QD layers. About $60 \%$ of this distortion arise from polaronic effects.

In summary, we found clear evidence for an optically induced tetragonal distortion of the GaAs crystal lattice into which (In,Ga)As QDs are embedded. The anisotropy of the distortion arises from polaronic effects initiated by QD confined carriers after their optical excitation. We believe that these studies are proof-of-principle studies and mark the beginning of x-ray studies of 'condensed matter systems in operation'. So far, condensed matter has been valuably studied by x-rays in passive mode, i.e. without excitation, to understand their structure on an atomistic scale. The impact of device operation on the lattice, especially under extreme excitation conditions, opens a new dimension of their understanding, and in particular, of the conditions that ultimately lead to their failure. Many further developments in the analysis of 
active devices can be foreseen such as higher spatial resolution along the vertical direction, angular dependence, or temporal resolution where, for example, the polaron formation dynamics in QDs is monitored.

We acknowledge the machine groups of HASYLAB and DELTA for providing synchrotron radiation. We also acknowledge support by the BMBF through grant no. 05K12PE1. S.T. and F. J. W. thank the NRW Forschungsschule 'Forschung mit Synchrotronstrahlung in den Nano- und Biowissenschaften' for financial support. J. M. (grant no. 05K10PEC) and A. D. W. (Wieck Q.com-H grant no. 16KIS0109) acknowledge financial support from BMBF. A.D.W. and M.T. thank the MERCUR foundation under contract number Pr-20130001 .

* Electronic address: sebastian.tiemeyer@tu-dortmund.de

† Present address: Optoelektronische Materialien und Bauelemente, Universität Paderborn, D-33098 Paderborn, Germany

[1] D. Bimberg, M. Grundmann, and N. N. Ledentsov, Quantum dot heterostructures, John Wiley \& Sons, Ltd.: New York (1998).

[2] Single Quantum Dots: Fundamentals, Applications and New Concepts, ed. by P. Michler, Topics in Applied Physics, Springer (2004); Single Semiconductor Quantum Dots (NanoScience and Technology), ed. by P. Michler, NanoScience and Technology, Spriner (2009).

[3] Quantum Dot Devices, ed. by Zhiming M. Wang, Lecture Notes in Nanoscale Science and Technology, Springer (2012).

[4] Semiconductor Quantum Bits, ed. by F. Henneberger and O. Benson, Pan Stanford Publishing (2009).

[5] A. Steinhoff, H. Kurtze, P. Gartner, M. Florian, D. Reuter, A.D. Wieck, M. Bayer, and F. Jahnke, Phys. Rev. B 88, 205309 (2013).

[6] S. Ates, S.M. Ulrich, A. Ulhaq, S. Reitzenstein, A. Löffler, S. Höfling, A. Forchel, and P. Michler, Nat. Photon. 3, 724 (2009).

[7] P. Borri, W. Langbein, S. Schneider, U. Woggon, R. L. Sellin, D. Ouyang, and D. Bimberg, Phys. Rev. Lett. 87, 157401 (2001).

[8] F.G.G. Hernandez, A. Greilich, F. Brito, M. Wiemann, D.R. Yakovlev, D. Reuter, A.D. Wieck, and M. Bayer, Phys. Rev. B 78, 17091 (2008).

[9] U. Bockelmann and G. Bastard, Phys. Rev. B 42, 8947 (1990).

[10] T. Inoshita and H. Sakaki, Phys. Rev. B 46, 7260 (1992).

[11] I. Vurgaftman, Y. Lam, and J. Singh, Phys. Rev. B 50, 14309 (1994).
[12] R. Ferreira and G. Bastard, Appl. Phys. Lett. 74, 2818 (1999).

[13] T. Inoshita and H. Sakaki, Phys. Rev. B 56, 4355 (1997).

[14] K. Kral and Z. Khas, Phys. Rev. B 57, 2061 (1998).

[15] O. Verzelen, R. Ferreira, G. Bastard, T. Inoshita, and H. Sakaki, phys. stat. sol. (a) 190, 213 (2002).

[16] S. Hameau, Y. Guldner, O. Verzelen, R. Ferreira, G. Bastard, J. Zeman, A. Lemaître, and J. M. Gérard, Phys. Rev. Lett. 83, 4152 (1999).

[17] H. Kurtze, J. Seebeck, P. Gartner, D. R. Yakovlev, D. Reuter, A.D. Wieck, M. Bayer, and F. Jahnke, Phys. Rev. B 80, 235319 (2009).

[18] X. D. Fan, T. Takagahara, J. E. Cunningham, and H. L. Wang, Solid State Commun. 108, 857 (1998).

[19] A. Vagov, V.M. Axt, and T. Kuhn, Phys. Rev. B 66, $165312(2002)$.

[20] P. Hawker, A. J. Kent, and M. Henini, Appl. Phys. Lett. 75, 3832 (1999).

[21] R. Bellingham, A. J. Kent, A. V. Akimov, and M. Henini, phys. stat. sol. (b) 224, 659 (2001).

[22] L. Goldstein, F. Glas, J. Y. Marzin, M. N. Charasse, and G. Le Roux, Appl. Phys. Lett. 47, 1099 (1985).

[23] J. Tersoff, C. Teichert, and M. G. Lagally, Phys. Rev. Lett. 76, 1675 (1996).

[24] C. Krywka, M. Paulus, C. Sternemann, M. Volmer, A. Remhof, G. Nowak, A. Nefedov, B. Pöter, M. Spiegel, and M. Tolan, J. Synchrotron Rad. 13, 8 (2006).

[25] O. H. Seeck, C. Deiter, K. Pflaum, F. Bertram, A. Beerlink, H. Franz, J. Horbach, J. Schulte-Schrepping, B. M. Murphy, M. Greve, and O. Magnussen, J. Synchrotron Rad. 19, 30 (2012).

[26] See, for example, F. Zamponi, P. Rothhardt, J. Stingl, M. Woerner, and T. Elsaesser, Proc. Natl. Acad. Sci. 109, 5207 (2012); C. v. Korff Schmising, M. Bargheer, M. Kiel, N. Zhavoronkov, M. Woerner, T. Elsaesser, I. Vrejoiu, D. Hesse, and M. Alexe, Phys. Rev. Lett. 98, 257601 (2007).

[27] J. Als-Nielsen and D. McMorrow, Elements of modern $x$ ray physics, John Wiley \& Sons, Ltd.: New York (2011), p. 221.

[28] A similar power level corresponds to a larger photon flux for the red illumination, while on the other hand the absorption coefficient is smaller than for the green laser. The net effect of these factors is a similar number of electron-hole pairs in the QDs for both excitation wavelengths, as evidenced from the photoluminescence spectra with comparable intensity in Fig. 1. Therefore, we expect comparable polaronic effects. Also the heating is likely comparable as both laser beams are completely absorbed in the crystals.

[29] P. W. Fry, I. E. Itskevich, D. J. Mowbray, M. S. Skolnick, J. J. Finley, J. A. Barker, E. P. O'Reilly, L. R. Wilson, I. A. Larkin, P. A. Maksym, M. Hopkinson, M. AlKhafaji, J.P. R. David, A. G. Cullis, G. Hill, and J. C. Clark, Phys. Rev. Lett. 84, 733 (2000). 